\title{
Tecnologías de la Información y la Comunicación en Educación Física: un análisis bibliométrico Information and Communication Technologies in Physical Education: bibliometric analysis \\ *Ana Isabel Sospedra Harding, *Paloma Escamilla Fajardo, **Sergio Aguado Berenguer *U niversidad Católica deVal encia (España), **U niversidad deValencia (España)
}

\begin{abstract}
Resumen. Influenciada por los cambios tecnológicos, sociales y culturales, la educación se ha visto obligada a integrar las Tecnologías de la Información y la Comunicación (TIC) en el aula. El mundo digital está en constante evolución, por lo que el profesorado necesita una formación continua para adaptar su metodología docente a los nuevos recursos tecnológicos en Educación Física (EF). Este análisis bibliométrico pretende identificar las líneas de investigación más activas en este campo de conocimiento hasta la fecha. La búsqueda ha sido realizada enW eb of Science Core Collection. Para ello se han analizado 155 artícul osy revisiones, de 349 autores de 30 países diferentes, publicados en 90 revistas. Según la red de co-pal abras, aparecieron 6 clusters, analizados temáticamente: (i) desar rollo de habilidades de uso de lasTIC en profesores y alumnos, (ii) ventajas de IasTIC en el ámbito del deporte, (iii) lucha contra la inactividad física a través de herramientas digitales, (iv) beneficios de la adquisición de habilidades relacionadas con lasT IC en la Educación Física, (v) profesores y alumnos que muestran una actitud positiva hacia la integración de lasTIC en la Educación Física, (vi) urgencia en el desarrollo de un nuevo sistema educativo regido por laigualdad de oportunidades Lainformación obtenidapuede contribuir aextender el conocimiento paraacadémicos y profesionales sobre el estado actual del campo de estudio. Del mismo modo, presenta lagunas existentes en la literatura que son necesarias de cubrir y se exponen futuras líneas o tendencias de estudio.
\end{abstract}

Palabras clave: Educación Física, Tecnologías de la Información y la Comunicación, TIC, análisis bibliométrico, actividad física.

\begin{abstract}
Influenced by technological, social and cultural changes, education has been forced to integrate Information and Communication Technologies (ICT) in the classroom. The digital world is in constant evolution, which means that teachers need continuous training in order to adapt their teaching methodology to the new technological resources in Physical Education (PE). This bibliometric analysis aims to identify the most active lines of research in this field of knowledge to date. The search was carried out inWeb of Science Core Collection. To this point, 155 articles and reviews have been analyzed, from 349 authors from 30 different countries, published in 90 journals. According to the network of co-words, 6 clusters appeared, analyzed thematically: (i) developing ICT use skills in teachers and students, (ii) advantages of ICT in the field of sport, (iii) combating physical inactivity through digital tools, (iv) benefits of acquiring ICT-related PE skills, (v) teachers and studentsshowing a positive attitude towardstheintegration of ICT in PE, (vi) urgency in the development of anew educational system governed by equal opportunities. The information obtained can contribute to extend knowledge for academics and professional s on the current state of the field of study. Likewise, it presents existing gaps in the literature that need to be filled and future lines or trends of study are presented.
\end{abstract}

Keywords: Physical Education, Information and CommunicationTechnologies, ICT, bibliometric analysis, physical activity.

\section{Introducción}

Las costumbres adoptadas por la cultura de la inmediatez y el mal uso de las tecnologías están jugando un papel muy importante a favor del sedentarismo, la obesidad y las enfermedades cardiovasculares (M eza y López, 2021). Por este motivo, en la actualidad se otorga una gran importancia social a la Educación Física(EF) debido al reconocimiento del papel decisivo que juega

Fecha recepción: 16-02-21. Fecha de aceptación: 08-03-21

Paloma Escamilla Fajardo

paloma.escamilla@uv.es en la adquisición de hábitos físicos y deportivos duraderos, junto con el bienestar y otras conductas saludables de los alumnos (León, Ayuso y Madrona, 2020; RamosEchazarreta et al., 2011).

Losrápidosavancestecnológicos experimentadospor la sociedad también se han integrado en el ámbito de la Educación Física. LasTecnologías de la Información y la Comunicación (TIC) representan algo más que el uso de un ordenador, ya que permiten, a través de diferentes dispositivos electrónicos, acceder a una variedad de fuentes de información que permiten mejorar sus conocimientos sobre la EF y sus conexiones y aplicaciones con otras áreas de aprendizaje (Stanescu et al., 2011). 
Además, las TIC ayudan a los estudiantes de EF a aprender promoviendo y mejorando sus habilidades, lo que les ayuda a seleccionar y aplicar habilidades tácticas eideas de composición. Según Prensky (2001), losalumnos son «nativos digitales» ya que han crecido con las TIC presentes en todos los ámbitos de su vida, pero esto no implica que todos los nativos digitales sepan ha cer un buen uso de las nuevas tecnologías (Briceño-Pira, Gómez \& Flórez, 2019). Por tanto, es responsabilidad del profesorado adaptar el uso de lasTIC a los objetivos de la asignatura. En este contexto, la implementación de las TIC en Educación Física permite la medición y evaluación del alumnado, facilitando la recogida de da tos por parte del profesor (Stanescu et al. , 2011). D ebido a su innegable importancia, ha surgido la motivación de investigar en detalle el origen y la evolución académica de lasTIC en la Educación Física.

\section{Educación Física}

En cuanto al contexto de la Educación Física, es el principal espacio de promoción para el desarrollo de las habilidades físicas y el fomento de la actividad física en niños y jóvenes. 0 frece una atención reglada por parte del profesorado para promover la actividad física, los conocimientos y los hábitos de vida sal udables de forma estructurada para todos los alumnos, en un entorno seguro (NASPE, 2005). En esta línea, Bailey (2006) tras revisar las evidencias científicas de los beneficios de la $E F$, concluyó que la EF puede tener un efecto positivo en los niveles físico, emocional, social y cognitivo, y en el estilo de vida. La calidad del programa de Educación Física es un factor determinante, ya que la adolescencia es una época de gran abandono deportivo, por lo que los profesores de Educación Física tienen la responsabilidad de acercar la actividad física a los alumnos y crear buenos hábitos deportivos (Castro et al., 2017; Gil, Cavichiolli, y Lucas, 2020).

En la misma línea, 0 kely et al. (2001) afirman en su investigación que hay pruebas de que las personas que han desarrollado una base sólida en habilidades de movimiento fundamentales tienen más probabilidades de ser físicamente activas, tanto durante la infancia como en la edad adulta. Por lo tanto, es importante motivar a los alumnos hacia la Educación Física y hacer que se sientan cómodos practicando deportes en la escuela. Si un alumno se siente satisfecho con las clases de Educa ción Física, crea un compromiso con ella (Elmore y Huebner, 2010). Y es bien sabido que un alumno comprometido con la asignatura crea vínculos más allá del ámbito escolar, lo que le lleva a tener hábitos de vida saludables en su vida joven y adulta (Git et al., 2020). Esto es importante porque el objetivo de la Educación Física no es sólo educar a los alumnos, sino también formar adultos responsables, activos y comprometidos.

\section{Tecnologías de la Información y la Comunica- ción (TIC) en Educación Física}

Los ordenadores personales pasar on a formar parte de las herramientas de las oficinas, los hogares y el ámbito de la educación a principios de la década de 1980. Esto planteó la cuestión de la necesidad de que los ciudadanos tuvieran conocimientos básicos y habilidades informáticas. En estecontexto, seañadió como unanueva asignatura al plan de estudios de las escuelas secundarias de N orteamérica y Europa del Este. «Veinte años después, la adquisición de conocimientos y habilidades bá sicas no parece ser suficiente para hacer frente a los cambiosen la sociedad» (Voogt \& Knezek, 2008, p. 118).

En este sentido, Kadam (2020, p. 178) define lasTIC como un «conjunto diverso de herramientas y recursos tecnológicos utilizados para comunicarse, y para crear, difundir, almacenar y gestionar información». En la actualidad, los estudiantes son considerados Nativos Digitales. Según la definición de Prensky (2001), los nativos digital es son la primera generación que ha crecido con las tecnologías digitales y son, por tanto, nativos del lenguaje propio de los ordenadores, los videojuegos e internet. Por el contrario, los inmigrantes digitales son aquellos que no han crecido en un mundo digital y han tenido que adaptarse a los cambios que ha generado la era digital. Este es un hecho que no puede pasar desapercibido en el aula, y por ello, los profesores, generalmente inmigrantes digitales, deben aprender a utilizar herramientas propias de esta nueva dimensión tecnológica para favorecer el proceso de enseñanza aprendizaje.

Desde el conocimiento científico, es necesario reflexionar sobre cómo ha evolucionado la práctica física, y cómo podemos promover la actividad física en Educa ción Física desde lasTIC. Según Stanescu et al. (2011, p. 6) «Como educadores no podemos ignorar la tecnología moderna y su tremendo impacto e influencia». Por ello, lasTIC deben integrarse en las sesiones educativas de forma fluida e interactiva, logrando efectivamente las competencias en todos los niveles. Esto debe implementarse siempre cuidando de no caer en la monotonía, ni en el conformismo de utilizar siempre las mismas herramientas, ya que a largo plazo pueden perder eficacia, sobre todo aquellas en las que el alumno interactúa. En la misma línea, Legrain et al. (2015, p. 
384) en su investigación sobre el impacto que el uso de lasTIC en las clases de Educación Física puede generar en la enseñanza, afirman que «la percepción de apoyo a la autonomía satisface las necesidades psicológicas básicas de los estudiantes».

El uso de medios audiovisuales en la asignatura de Educación Física representa un sistema moderno que permite un aprendizaje más rápido de los ejercicios físicos (Stanescu et al, 2011). Existen multitud de recursos para integrar lasTIC en el aula de Educación Física, en todos los niveles, lo que no implica que se reduzca la actividad física, sino todo lo contrario, estas nuevas herramientas deben invitar a los alumnos a ampliar sus conocimientos y mejorar sus habilidades motrices. Por ello, los profesionales del mundo de la actividad física están mostrando interés en construir entornos tecnológicos digitales estructurados y significativos para aprovechar la propensión de los al umnos a utilizar sus dispositivos durante su tiempo libre. Uno de los principales objetivos de la integración de las TIC en los programas de promoción de la actividad física es motivar a los adolescentes y jóvenes para que sean más activos físicamente (NASPE, 2004).

En el ámbito del deporte, una acción motriz depende en gran medida de la percepción del movimiento. Por lo tanto, proporcionar a los estudiantes datos sobre las características clave de la capacidad de ejecución (por ejemplo, el ritmo, la dirección, el golpeo del balón), permite a los estudiantes integrar la nueva información, a su propio ritmo, lo que en última instancia conduce a los beneficios motores (Beashel y Sibson, 2000; Legrain et al., 2015). Sin embargo, las IC nunca deben sustituir la acción motriz ni reducir el tiempo de compromiso motor (Filgueira-Gomis, 2016), sino que deben ser un medio para fomentar la actividad física.

Existen evidencias de las múltiples ventajas del uso de internet como medio para realizar actividad física, a través de aplicaciones en dispositivos digitales. Entre ellas, destaca la accesibilidad y que se ajustan a las necesidades del usuario, son capaces de proporcionarle indicaciones y feedback per sonalizados. Además, las apps son potentes y tienen múltiples funciones interactivas (Middelweerd et al., 2014). La portabilidad de los teléfonos móviles permite a los usuarios tener acceso las 24 horas del día, lo que posibilita la gestión a largo plazo y refuerza los hábitos de vida saludables a través de diversas comunicaciones y aplicaciones (Flores et al., 2015). Del mismo modo, al alumnado con necesidades puede beneficiarle el impacto de las TIC en Educación Física (Batanero et al., 2020).
Es interesante integrar recursos digitales potentes en la asignatura de Educación Física, para mostrar las diferentes herramientas que se pueden utilizar tanto dentro como fuera del aula para mantenerse físicamente activo. Asimismo, los profesores pueden enseñar a Ios alumnos a hacer un uso responsable de estas aplicaciones y mostrar las claves para mantener una actitud crítica y favorable a la hora de elegir una app u otra. La implantación de las TIC en la escuela puede motivar a los alumnos a la práctica del deporte, generar hábitos de vida saludables y combatir así al gunos problemas sociales importantes como la obesidad y el sedentarismo en los jóvenes.

Sin embargo, a pesar de su creciente importancia e interés, no existe un análisis macroscópico de la litera tura existente sobre lasTIC en la Educación Física. Por esta razón, el presente estudio bibliométrico es importante y tiene los siguientes objetivos generales (i) Conocer el estado de las TIC en Educación Física a partir de la literatura existente. De este objetivo general se derivan los siguientes objetivos específicos: (a) Identificar a los autores, revistas, instituciones y países más importantes en el campo de estudio. El segundo objetivo general es (ii) anal izar cronológicamente la literatura existente sobre las TIC en Educación Física, y (iii) explorar las áreas temáticas existentes sobre la implementación de lasTIC en Educación Física. De este último objetivo general se pueden proponer dos objetivos específicos: (b) realizar un análisis temático de cada una de las áreas temáticas, (c) proponer huecos en la literatura y futuras líneas de investigación.

\section{Método}

Con el tiempo, surge la necesidad de evaluar el desempeño de la actividad científica y su impacto en la sociedad. Por ello, a través de los controles de calidad en el mundo de las publicaciones científicas, es necesa rio controlar el valor de lo que se publica, y con esta función se aplican los indicadores bibliométricos (Skute, 2019).

Los datos se han extraído de laWeb of Science Core Collection, incluyendo la base de datos del Institute for Scientific Information (ISI) deFiladelfia: Science Citation Index (SCI), Social Science Citation Index (SSCI) yArts and Humanities Citation Index (AyHCl) como han hecho autores anteriores (Escamilla-Fajardo, Núñez-Pomar, Ratten \& Crespo, 2020). La búsqueda se ha realizado en el campo temático a través de la siguiente ecuación de búsqueda: TS= [(«information and 
communication technolog*» $0 \mathrm{R}$ «new technolog*») AND («physical education»)], obteniendo 155 documentos: 153 artículos y 2 revisiones. La búsqueda se realizó en inglés, ya que la mayor parte de la literatura académica está en este idioma. Para obtener los datos de este análisis bibliométrico se han utilizado criterios de selección por año de publicación, fijándolo hasta el 31 de diciembre de2020, paratener información hastaun año. También se utilizó como criterio de inclusión que fuesen artículos y revisiones, excluyendo cualquier otro tipo de documento. No se han utilizado más criterios de selección inicial.

Todos los registros se han descargado en formato de texto plano (txt) con los siguientes campos: autores, palabras clave, año de publicación, categoría temática, nombre de la publicación, ISSN (International Standard Serial Number) y veces citadas.

Losprogramas estadísticosutilizadosfueron HistCite, para analizar y organizar los resultados de una búsqueda. Bibexcel para la manipulación y transformación de registros bibliográficos. Por último, Vo Sviewer para la representación y el análisis de la información.

\section{Resultados}

A continuación, se presentan los resultados obtenidos tras el anál isis bibliográfico de lasTIC y laEF Inicialmente, se presentan los indicadores del análisis bibliométrico básico (año de publicación, autores, país, institución, revistas, idiomas y palabras), seguidos de las redes de co-ocurrencia (co-palabras) y el análisis temá tico.

\section{D atos bibliométricos básicos}

El número de artículos analizados asciende a 155, publicados en 90 revistas, escritos por 349 autores de 171 instituciones de 30 países. En esta sección se pre-

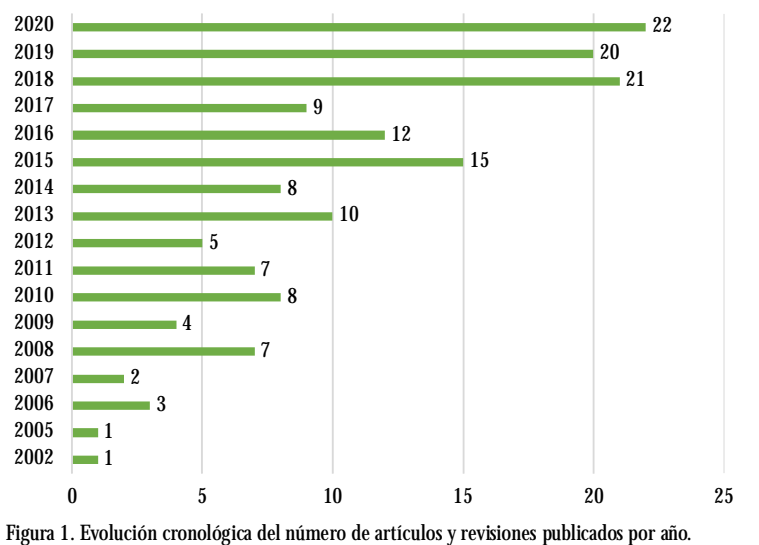

sentan indicadores relacionados con los años de publica ción y los autores, revistas, instituciones y países más productivos. La figura 1 muestra el número de artículos publicados por año desde 2002 hasta2020. En 2015 se refleja un aumento, se registraron hasta 15 publica ciones científicas. El año con mayor número de artícuIos publicados sobre este tema fue el 2020, que muestra el valor más alto con un total de 22, precedido por el 2018 y el 2019 en los que se publicaron un total de 21 y 20 artículos y revisiones respectivamente.

En cuanto a los autores, 349 investigadores publica ron al menos un artículo sobre este tema. Entre ellos, los investigadores que más artículos publicaron fueron M. Papastergiou con 6 artículos, A. Bæena-Extremera, A. Casey y O.C. Foguet con 4 artículos cada uno, y F. Zurita-O rtega con 3 artículos. El autor con mayor número de citas (TGCS) es M. Papastergiou (TGCS= 78). La tabla 1 muestra los autores ordenados por el número de publicaciones, destacando los que han sido más productivos.

\begin{tabular}{|c|c|c|c|c|c|}
\hline Autores & Afiliación & & Artículos & TLCS & TGCS \\
\hline M. Papastergiou & University ofT hessaly & & 6 & 14 & 78 \\
\hline A. Bæena-Extremera & U niver sidad de Granada & & 4 & 0 & 3 \\
\hline A. Casey & Loughborough University & & 4 & 5 & 51 \\
\hline O.C. Foguet & $\begin{array}{l}\text { Institut Nacional d'Educació } \\
\text { Catalunya }\end{array}$ & Física de & 4 & 0 & 0 \\
\hline $\begin{array}{l}\text { F. Zurita- } 0 \text { rtega } \\
30 \text { autores }\end{array}$ & U niver sidad de Granada & & $\begin{array}{l}3 \\
2\end{array}$ & $\begin{array}{l}0 \\
-\end{array}$ & $\begin{array}{l}10 \\
-\end{array}$ \\
\hline 314 autores & . & & 1 & - & - \\
\hline
\end{tabular}

En relación con los países de los autores menciona dos, investigadores de 30 países diferentes publicaron al menos un artículo sobre lasTecnologías de la Informa ción y la Comunicación en la Educación Física (Tabla 2). Lamayoríade las publicaciones proceden deEspaña(70), precedidas por el Reino Unido (12), que ha recibido el mayor número de citas tanto en nuestra búsqueda (TGCS= 32) como en la base de datos global (TGCS= 186). La tabla2, muestra los países que han publicado el mayor número de artículos relacionados con este tema.

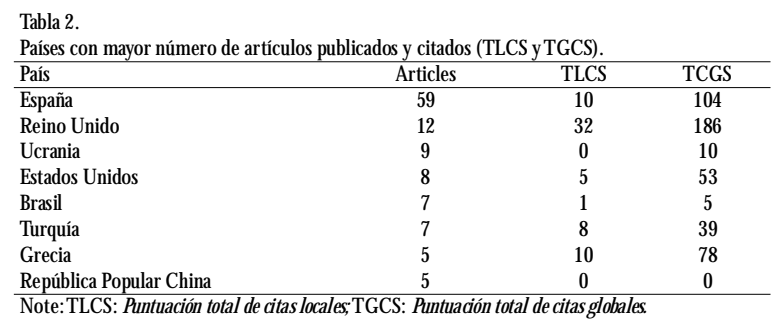

Los 155 artículos y reseñas publicados sobreTecnologías de la Información y la Comunicación en la Educa ción Física procedían de 171 instituciones diferentes. En 17 de los artículos algunos de los autores eran de la 
Universidad de Granada, en nueve de la Universidad de Sevilla, en ocho de la Universidad de Murcia y en seis de la U niversidad deTesalónica. La tabla 3, muestra las instituciones con mayor número de artículos publicados relacionados con este tema.

Tabla 3.

Instituciones con mayor número de artículos publicados y el número de veces que han sido citados (TLCSYTGCS).

citados (TLCS

Institución

Universidad de Sevilla

Univer sidad de M urcia

Universidad deTesalónica

Universidad de Barcelon

Univer sidad deValencia

Universidad deAlmería

Universidad de Lleida

Universidad de Santiago de Compostel

Note: TLCS: Puntuación total de citas locales; TGCS: Puntuación total de citas globales

Un total de 90 revistas han publicado al menos un artículo específico sobre Educación Física y Tecnologías de la Información y la Comunicación. Como se puede observar en laTabla 4, de todas las revistas, Retos- Nuevas tendencias en Educación Física, deporte y recreación destaca como la revista con mayor número de artículos publicados sobre este tema (18), aun así, no es la que mayor número de citas ha recibido (14). En segundo lugar, la revista que más artículos ha publicado sobre este tema en particular es Pedagogics Psychology Medical. Problemas biológicos del entrenamiento físico y el deporte con 7 publicaciones, revista que ha recibido un total de 8 citas en la base de datos global. En tercer lugar, Apunts. Educación Física y Deportes, no ha recibido ninguna cita en WoS, pero es la tercera revista en número de publicaciones (5).

\begin{tabular}{|c|c|c|c|}
\hline Revista & Artículos & TLCS & TGCS \\
\hline Retos. Nuevas tendencias en educación física, deporte y recreación. & 12 & 2 & 13 \\
\hline $\begin{array}{l}\text { Pedagogics psychology medical. Biological problems of physical } \\
\text { training and sports }\end{array}$ & 7 & 0 & 8 \\
\hline Apunts Educación Física y Deportes & 5 & 0 & 0 \\
\hline European Physical Education Review & 4 & 1 & 20 \\
\hline 8 revistas con 3 artícul os y/o reseñas & 24 & & \\
\hline 12 revistas con 2 artícul os y/ o reseñas & 24 & - & - \\
\hline 58 revistas con 1 artícul o y/ o reseña & 58 & - & - \\
\hline
\end{tabular}

La tabla 5 muestra, de mayor a menor, el número de citas de artículos relacionados con este tema. La revistamás citada es British Journal of EducationalTechnology, citada en nuestra búsqueda 19 veces (TLCS= 19; TGCS $=80$ ). La segunda revista más citada es Computers and Education, citada 9 veces en nuestrabúsqueda(TLCS=

\begin{tabular}{|c|c|c|c|}
\hline Revista & Artículos & TLCS & TGCS \\
\hline British Journal of educational technology & 2 & 19 & 80 \\
\hline Computers and education & 2 & 9 & 60 \\
\hline European Journal of teacher education & 1 & 7 & 32 \\
\hline Technology pedagogy and education & 2 & 4 & 29 \\
\hline Sport, education and technology & 3 & 0 & 26 \\
\hline Journal of physical education, recreation and dance & 3 & 1 & 24 \\
\hline European physical education review & 4 & 1 & 20 \\
\hline Journal teaching physical education & 2 & 4 & 20 \\
\hline
\end{tabular}

9) y 60 citas en laWoS (TGCS= 60). La tercera revista más citada es European Journal of Teacher Education, que ha sido citada localmente 7 veces y globalmente 32 veces $(\mathrm{TLCS}=7 ; \mathrm{TGCS}=32)$.

\section{Referencias más citadas}

Los 155 artículos analizados han utilizado 4.744 referencias entre las que destacan: el artículo deThomas y Stratton (2006) referenciado en 19 publicaciones de la búsqueda, en segundo lugar, el artículo de Ambrós et al. (2013) referenciado en 16 publicaciones de la búsqueda, y con 9 referencias encontramos el artículo de Kretschmann (2015) (Tabla 6).

Tabla 6 .

Artículos más referenciados (=8)

Referencias

Thomas y Stratton (2006)

Ambrós et al. (2013)

Kretschmann (2015)

Papastergiou (2009)

Prensky (2001)

Tearle y Golder (2008)

\section{Análisis de co-ocurrencias}

Esta sección analiza la red de co-ocurrencia de pala bras clave. Según Guan, Gao, Zhang y Zhou (2018), el análisis de co-palabras tiene lugar cuando dos términos aparecen simultáneamente en un texto. El objetivo es averiguar la estructura que conforma el dominio científico. Tras seleccionar los términos, se calculan las medidas de similitud para concluir con una agrupación por áreas temáticas. Sobre la red bibliométrica generada se han aplicado medidas de similitud, para la normalización de los valores de co-ocurrencia de las palabras clave, y técnicas de clustering multivariante para identificar grupos homogéneos de palabras clave.

Para llevar a cabo este análisis, se han seleccionado dos tipos de palabras clave, otorgadas por los propios autores para definir el contenido de sus documentos, conocidas como Keyword Author (KWA); y KeyWords Plus (KW +), palabras clave extraídas automáticamente a partir de la frecuencia de aparición de las palabras en los títulos de las referencias de los artículos (Gao, Zhao, $\& \mathrm{Li}, 2019)$. Ambos registros se presentan en este trabajo.

En los 155 artículos y revisiones que tratan sobre la Educación Física y lasTecnologías de la Información y la Comunicación, se han registrado 530 palabras, de las cuales las más utilizadas fueron «Physical Education» (219), «technolog*» (44), «TIC»(31), «aprender*»(29), «alumno*» (27), y «enseñar*» (45).

Como puede verse en la Figura 2, en el análisis de las relaciones entre las palabras clave, dos clusters formados por 12 términos cada uno (cluster rojo y cluster 
verde) destacan sobre el resto. En el clúster rojo, el término que ha aparecido con mayor frecuencia ha sido «tecnología», término que aparece como núcleo de esta gran red de términos. En el clúster verde, la palabra que más ha aparecido en los artículos ha sido «TIC», que también aparece como núcleo de esta red de términos. Estos clusters están precedidos por otras dos redes de 8 términos cadauna (cluster azul oscuro y cluster amarillo). La palabra con mayor coocurrencia en el clúster azul oscuro ha sido «salud», que es el núcleo de la red. La palabra más frecuente en el clúster amarillo de la figura 4 ha sido «Educación Física». Las siguientes palabras más utilizadas han sido «actitudes» e «informa ción y comunicación».

Los términos con mayor co-ocurrencia se ilustran en la Figura 2 con un tamaño mayor que el resto de los nodos. Teniendo en cuenta esto, cabe destacar la rela ción entre las palabras clave «Educación Física», «TIC» y «tecnología», ya que éstas actúan como enlace con las demás palabras clave.

oscuro, amarillo, morado y azul claro).

A continuación, se presentan los datos con más deta lle, clasificados por redes de enlaces entre las palabras clave y su co-ocurrencia.

Cluster rojo - «Tecnología», «lnformación» y «Estudiantes»; este grupo está formado por doce términos: adolescentes, creencias, ordenador, juegos, género, información, internet, motivación, software, estudiantes, apoyo, tecnología. Cuya temática está relacionada con el rápido desarrollo de la tecnología digital y el impacto que la óptima integración de lasTIC puede tener en la comunidad educativa, especialmente en la asignatura de Educación Física.

«El desfase entre la práctica en el aula y la formación en la educación superior requiere una transformación del modelo académico» (Hinojo-Lucenaet al, 2018, p. 1). El cambio debe real izarse sobre una base educativa sólida y creciente. La integración de nuevas herra mientas y metodologías supone un reto a lo que comúnmente se conoce como enseñanza tradicional, que ha permanecido en el sistema educativo durante décadas. Los estudiantes digitales tienen necesidades que deben ser atendidas con herra mientas de aprendizaje a las demandas de la sociedad actual. Por ejemplo, con el uso del «flipped learning» en Educación Física(Hinojo-Lucenaet al., 2020). A lo largo de diferentes estudios de investigación, se haconstatado que tanto los profesores como los alumnos muestran actitudes positivas en la

\section{Análisis temático}

En el análisis temático se han utilizado 48 términos diferentes, que se han agrupado por clústeres (Figura 2). Un cluster es un conjunto de elementos incluidos en un mapa de conocimiento. Los clusters no se solapan en Vo Sviewer, por lo que un elemento puede pertenecer a un solo cluster. Así, se han identificado seis pequeños grupos entre 2002-2020, donde cada término se ha asociado en un cluster diferenciado por colores y líneas según el cluster al que pertenecen (rojo, verde, azul integración de las tecnologías para la transmisión de conocimientos, y mejoras significativas en el desarrollo de lasTIC (Goktas, 2012; Papastergiou, 2010). Existen evidencias científicas que demuestran los múltiples beneficios del uso de las Tecnologías de la Información, como herramienta potencial en beneficio de las competencias curriculares de los al umnos. Entre ellos, destaca el mayor compromiso y autonomía de los alumnos durante el proceso de enseñanza y aprendizaje. Por otro lado, una gestión y organización más eficiente del apren- 
dizaje (Per rota, 2013). Sin embargo, los educadores no solo deben mantener una formación continua para desarrollar las habilidades de uso de las TIC, sino también para introducirlas en las clases de Educación Física de forma efectiva en su práctica diaria (Koekoek et al, 2018).

Sin embargo, la realidad es que el rápido desarrollo de la tecnología digital amplía las perspectivas de su aplicación en los programas de enseñanza de la Educación Física. Pero a menudo, los educadores, interesados en la integración de las TIC, no están suficientemente equipados para integrar eficazmente los recursos tecnológicos (Koekoek et al., 2018). 0 bien carecen de los conocimientos prácticos necesarios para potenciar las herramientas digitales, y se aplican de forma incorrecta (Göktå, 2012).

Cluster verde - «TIC» y «Deporte»; compuesto por once términos: competencia digital, TIC, impacto, innovación, instrucción, metodología, dispositivos móviles, rendimiento, jugadores, deporte, deportes. El tema de este grupo está relacionado con las ventajas de Ias TIC en el ámbito del deporte a través de los videojuegos y las aplicaciones.

Los profesores de Educación Física y los profesiona les del deporte consideran el uso de las TIC como un recurso potencial para mejorar la calidad de la enseñanza. Sin embargo, la realidad dista mucho de las expecta tivas deseadas. La literatura científica refleja una grave escasez de desarrollo profesional específico para la asignatura de Educación Física (Tearle \& Golder, 2008), la paridad técnica y formativa no es habitual en el sistema educativo debido, principalmente, a un desconocimiento generalizado de las necesidades y exigencias que requiere esta aplicación digital.

Sin embargo, los profesionales de laFP y de las Ciencias del Deporte reconocen que las TIC son un componente integral delaformación curricular (Göktas, 2012; Papastergiou, 2010), y el desarrollo de las competencias en TIC es fundamental para mejorar las actitudes hacia el mundo digital e Internet (Papastergiou, 2010).

Clúster azul oscuro - «Salud» y «Escuela»; compuesto por ocho términos: niños, educación, salud, nuevas tecnologías, percepciones, actividad física, escuelay videojuegos. Este clúster sugiere que las escuelas traba jen con bloques de contenidos de Educación Física a tra vés de herramientas digitales (TIC), con el fin de combatir la inactividad física, como los videojuegos activos (conocidos como exergames).

La integración de innovaciones tecnológicas aplica bles en los centros escolares, con el fin de reducir las cifras registradas de inactividad física provocadas por el sedentarismo de la pantalla digital, que es una de las principales causas de los altos niveles de obesidad en la sociedad actual (Panadero-Pérez, Román, Joya-Barroso \& Román-Mata, 2019). LasTIC han propiciado procesos de enseñanza y aprendizaje más flexibles y significa tivos (Zurita-O rtega, Chacón-Cuberos, Castro-Sánchez, Gutiér rez-Vela, \& González-Valero, 2018). Asimismo, la introducción de lasTIC en laEducación Física mejora las formas organizativas de la formación de maestros y profesores altamente cualificados, e intensifica las actividades deportivas y recreativas entre los miembros de la sociedad educativa (Chukhlantseva, 2017). Los videojuegos activos y los juegos motores son un recurso motivador para seguir un estilo de vida activo (Cuberos, Sánchez, Ortega, Garcés, \& Martínez, 2016). Requieren fuerza, coordinación y flexibilidad, habilidades que se integran en los programas de Educación Física en los países desarrollados. Los exergames (ejercicios y juegos) promueven el desarrollo físico, psicológico e intelectual de los estudiantes y los animan a ser físicamente activos a través del desarrollo de la motivación (Chukhlantseva, 2017). Por ello, se considera una herramienta a tener en cuenta para hacer ejercicio en edad escolar y promover un aprendizaje más significa tivo.

Clúster Amarillo - «Educación Física» e «innovación educativa»; compuesto por ocho términos: innova ción educativa, tecnología educativa, educación superior, pedagogía, Educación Física, formación del profesorado, enseñanza, universidad, que hacen referencia a los beneficios relacionados con la adquisición de lascompetencias docentes relacionadas con las TIC de Educación Física, con el objetivo de aumentar la motivación e implicación del alumnado.

La literatura científica revela que, a través de la adquisición de competencias docentes relacionadas con las TIC, se incrementa la motivación e implicación del alumnado, mediante estrategias de gamificación, TIC y evaluación continua en el aula (C astañeda-Vázquez, Espejo-Garcés, Zurita-O rtega, \& Fernández-Revelles, 2019). Por ello, se considera oportuno asumir la innovación como un componente integral de las profesiones curriculares de EF. Dando a los estudiantes la oportunidad de obtener habilidades digitales a través de la actividad física.

Por otro lado, encontramos artículos que ponen de manifiesto la falta de formación profesional del profesorado, y su desconocimiento y comprensión de la necesidad de utilizar las TIC (Tearle \& Golder, 2008) como 
recurso integrador y necesario para que los alumnos puedan integrarse en el mundo laboral una vez finaliza dos sus estudios. La falta de comprensión de las necesidades, desalienta la implementación de un modelo innovador de enseñanza y aprendizaje en los departamentos escolares de Educación Física.

Cluster morado - «Actitudesy autoeficacia»; compuesto por seis palabras clave: actitudes, aula, contenido pedagógico, conocimiento, autoeficacia, formación del profesorado, TPACK (technological pedagogical content knowledge). Se refiere a que todos los miembros de la comunidad educativa muestran una actitud positiva hacia la implementación de lasTIC en el proceso de enseñanza y aprendizaje de la Educación Física. Podemos observar dos enfoques: por un lado, encontramos estudios que analizan las actitudes y la formación del profesorado en el uso de lasTIC en el proceso de enseñanza y aprendizaje. Por otro lado, encontramos artículos que analizan la cantidad de herramientas digitales de las que disponen los centros educativos para la óptima transmisión de conocimientos y para alcanzar las competencias curriculares necesarias.

Existen efectos directos significativos de las actitudes de los profesores en formación hacia el uso de la tecnología, las competencias tecnológicas y las experiencias sobre sus creencias de autoeficacia TPACK. (Yederlen-Damar, Boz y Aydin-Günbatar, 2017). La mayoría de los profesores de Educación Física consideran que se trata de una valiosa herramienta de enseñanza (Göktå, 2012). Los estudiantes aprecian plenamente la omnipresencia de lasTIC (Papastergiou, 2010). EI uso de dispositivos digitales no solo beneficia las actitudes y la transmisión de conocimientos y valores, sino que también contribuye favorablemente al desarrollo de las habilidades tanto motrices como digitales

Clúster Azul Claro - «Tecnología de la Informa ción y la Comunicación»; Compuesto por tres términos: tecnología de la información y la comunicación, innovación docente, webquest. Se refieren a diferentes herramientas digitales. Los artículos que forman parte de este clúster hacen referencia a los cambios en el contexto educativo actual, descritos y afectados por los nuevos modelos de familia profesional, por los nuevos entornos y por la diversidad superlativa del alumnado, provocando la urgencia de desarrollar un nuevo sistema educativo que se rija por el principio de igualdad de oportunidades y no discriminación (Abuin, 2008). Para la búsqueda y transformación del sistema educativo actual, lasTIC asumen un papel indispensable en las instituciones educativas. Y la EP no puede quedarse al mar- gen ni permanecer indiferente ante el amplio abanico de posibilidades que ofrecen lasTIC.

\section{Discusión}

El desarrollo de la tecnología digital amplía las perspectivas de su aplicación en los programas educativos de Educación Física. Sin embargo, la literatura existente muestra que existen múltiples elementos que impiden 0 no favorecen la integración de lasTIC en el proceso de enseñanza-aprendizaje. Por ello, su estudio y análisis desde una perspectiva educativa es de vital importancia para conocer el estado de las TIC en Educa ción Física. Este ha sido uno de los objetivos que pretendía el presente estudio, aportar información interesante y actualizada sobre el estado del conocimiento. En base a ello, se han identificado los principales autores que han estudiado y publicado sobre las TIC en este ámbito de actuación, las revistas más importantes en las que se han publicado, así como las instituciones y páses que han aportado en mayor medida a este campo. El nivel de cumplimiento de este objetivo general y objetivo específico ha sido elevado.

En este sentido, las instituciones deben asegurar los medios, la accesibilidad de sus disposiciones y su respectivo desarrollo, para que los docentes obtengan las competencias necesarias para integrar la tecnología en la enseñanza de manera efectiva. Por lo tanto, es necesario que las políticas públicas y gubernamentales brinden oportunidades para que esto sea posible (Wyant \& Baek, 2019).

En relación con la influencia de las medidas institucionales y la competencia tecnológica en la enseñanza, Legrain et al. (2015), en su investigación, muestran la falta generalizada de incentivos para los profesores, tanto a nivel económico como académico, así como de carga lectiva. Esta puede ser una de las razones por las que la implementación de las nuevas tecnologías en la educación es lenta y engorrosa.

Considerando el segundo objetivo general, se ha realizado un análisis cronológico de la literatura existente sobre las TIC en Educación Física. De ahí se ha podido extraer que es todavía un área de estudio que Ilama la atención de académicos y profesionales al continuar con la tendencia ascendente en cuanto al número de publicaciones. Esto quiere decir que es un campo nicho y que tiene todavía posibilidades de aportaciones y consideraciones importantes.

En este sentido, Teo (2008) afirma que el uso de las TIC en el aula depende en gran medida de la actitud de 
los profesores hacia la tecnología, y que con el tiempo estos están considerando en mayor medida su inclusión. La formación del profesorado debe ser ágil y continua para cambiar las prácticas docentes en el aula. Deben responder a las necesidades de los al umnos que se consideran nativos digitales y formarlos para que puedan afrontar con éxito las exigencias de la sociedad actual. La actitud de los profesores, general mente inmigrantes digitales, y su disposición a utilizar las TIC es clave para la transformación del modelo académico. Sin embargo, hay que destacar que para que esto se produzca de forma efectiva, debe haber una formación previa y generalizada en el uso adecuado de lasTIC en la educación.

Tanto la administración como el profesorado deben tener en cuenta que, si quieren reducir los índices de obesidad en la sociedad y reducir el número de abandonos escolares prematuros y mejorar la calidad de vida de las futuras generaciones, hay que asumir ciertas responsabilidades. Las instituciones y la asignatura de Educación Física pueden hacer mucho. U na de las mejores formas de intervenir desde la educación es a través de propuestas e intervenciones que pretendan fomentar el uso de lasTIC como herramienta para la adquisición de hábitos de vida sal udables, el mantenimiento de una vida activa, y la utilización de recursos digitales como elemento para mantener la motivación y el interés por formar parte de la comunidad educativa.

Por último, el tercer objetivo principal está relacionado con la exploración de las áreas temáticas principa les. En este sentido se presentan dos objetivos específicosque han sido cumplidos en gran medida. Por un lado, se han propuesto seis áreas temáticas que han sido deta lladas en el apartado anterior. De ahí se puede extraer la tendencia que tiene la literatura existente hacia la exploración y el estudio. También cada uno de los autores que han focalizado su interés y effuerzo en ampliar esa área de estudio. Por último, proponer huecos existentes en la literatura y futuras líneas de investigación interesantes para rellenar estos huecos y aportar información novedosa y útil.

\section{Conclusiones}

Este estudio ofrece una visión macroscópica de la evolución de las TIC en la Educación Física hasta el 31 de diciembre de 2020. El estudio incluye 155 publica ciones (153 artículos y 2 revisiones), publicadas por 349 autores de 171 instituciones y 30 países. Todas ellas han sido publicadas en un total de 90 revistas científicas. Se trata del primer estudio sobre este tema en un ámbito tan específico como el de la Educación Física.

Los resultados obtenidos en este estudio permiten conocer mejor el desarrollo de lasTIC en la Educación Física. Proporciona datos relevantes y también una visión general de los principales autores, la evolución cronológica de las publicaciones, las principales revistas en las que se han publicado y un análisis temático que ayuda a comprender mejor las subáreas del campo de estudio. Q uizás este análisis temático por clústeres sea una de las mayores aportaciones al ayudar a los académicos y profesional es a identificar los temas de estudio. Las principales áreas engloban diferentes aspectos rela cionados con las TIC en la Educación Física. En primer lugar, destaca el área relacionada con el creciente y continuo desarrollo de la tecnología y la integración de las TIC en la comunidad educativa, concretamente en la Educación Física. En segundo lugar, destaca un área reIacionado con las ventajas que tienen lasTIC en el ámbito deportivo, considerando aplicaciones y videojuegos. En tercer lugar, se centra en los exergames y el uso de herramientas digitales en el trabajo de los contenidos de Educación Física. En cuarto lugar, esta área está rela cionada con la formación y adquisición de competencias digitales por parte de los docentes de Educación Física, y qué impacto puede tener en el alumnado. En quinto lugar, esta área está relacionada con la actitud positiva de todos los miembros de la comunidad educativa hacia lasTIC en Educación Física. Por último, la última área engloba herramientas digitales y hace referencia a los nuevos entornos educativos en los que debe destacar el respeto, la igualdad y la no discriminación teniendo un papel destacado lasTIC.

Además, esta investigación ha revelado varias lagunas o vacíos en la literatura sobre la aplicación y desarrollo de lasTIC en la Educación Física. Se ha observado que se trata de un campo nicho, ya que es relativamente reciente y está en continuo estudio y desarrollo. Muchos autores han centrado su atención en él en los últimos años de forma teórica y empírica. Sin embargo, de forma común, todos los grupos analizados convergen en la necesidad de introducir y adaptar las TIC a la educación, intentando motivar a los alumnos y enseñar un buen uso de las mismas en el aula. La actividad física puede ser fomentada a través de las TIC y esto debe ser explorado y aprovechado por los profesores de Educación Física.

No obstante, este estudio tiene varias limitaciones que no deben ser ignoradas. En primer lugar, la búsqueda se ha realizado en una única base de datos (Web of Science Core Collection), y aunque se considera una 
fuente fiable de documentos de alto impacto (Yang et al. , 2013), es posible que no se haya estudiado información relevante. Por otro lado, la búsqueda se ha realiza do con términos en inglés ya que es el idioma más utilizado en el ámbito académico, sin embargo, puede haber condicionado la búsqueda de información relevante. Por último, la búsqueda se ha realizado hasta el año 2020 para tener la información de años completos, sin embargo, esto podría haber perdido información de artículos recientes publicados en 2021 que no se han tenido en cuenta.

Por último, se considera interesante para futuras investigaciones señalar los diferentes beneficios de la Educación Física y las ventajas que ofrece la integración de lasTIC en la misma. Una revisión sistemática puede ser un futuro trabajo de revisión que podría aportar información relevante y más concisa sobre el área de estudio. LasTIC deben de ser consideradas como una oportunidad, en lugar de como un problema. Por ello, se invita a analizar y señalar las limitaciones existentes para la aplicación y uso de lasTIC en Educación Física. No obstante, cabe destacar que no se deben de dejar de lado las comunidades aisladas, teniendo en cuenta consideraciones realistas sobre las restricciones de los colectivos más pobres, evitando la brecha educativa y ofreciendo igualdad de oportunidades a toda la comunidad educativa.

\section{References}

Ambrós, Q., Foguet, O., \& Rodríguez, J. (2013). Introducción de IasT IC en Educación Física. Estudio descriptivo sobrelasitua ción actual. Apunts. Educación Física y Deportes 2(3), 37-44. https:/ / doi.org/ 10.5672/ apunts. 2014-0983.es. (2013/ 3). 113.03

Bailey, R. (2006). Physical education and sport in schools: A review of benefitsand outcomes. Journal of School H ealth, 76(8), 397401. https/ / doi.org/10.1111/ j.1746-1561.2006.00132.x

Batanero, J. M. F., Rueda, M. M., Cerero, J. F., \&Tadeu, P. Impacto de lasTIC en el alumnado con discapacidad en el área de Educación Física: una revisión sistemática. Retos, nuevastendenciasen educación física, deportey recreación , (39), 849 - 856. https:/ / doi.org/ 10.47197/ retos. v0i39. 78602

Beashell, P., \& Sibson, A. (2000). ICT in Physical Education-Help or Hindrance?British Journal ofTeaching Physical Education,31(2), 6-9.

Briceño-Pira, L., Muñoz, D. P. G. \& \& Romero, R. F. (2019). Usosde IasTICsen preescolar: hacialaintegración curricular. (U ses of ICT in preschool: towards curricular integration). Panorama, 13(24), 20-32. https:/ / doi.org/ 10.15765/ pnrm.v13i24.1203

Castañeda-Vázquez, C., Espejo-Garcés, T., Zurita-O rtega, F., \&
Fernández-Revelles, A. B. (2019). Laformación delosfuturos docentesatravésdelagamificación, ticy evaluación continua. SPORT TK-Revista EuroAmericana de Ciencias D el D eporte, 8(2), 55-63. https:/ / doi.org/ 10.6018/ sportk. 391751

Castro-Sánchez, M ., Linares M anrique, M., Sanromán-M ata, S., \& Pérez-Cortés,A.J. (2017). Análisis delos comportamientos sedentarios, prácticade actividad físicay uso de videojuegosen adolescentes. Sportis, 3(2), 241-255. https:/ / doi.org/ 10.17979/sportis.2017.3.2.1746

Chukhlantseva, N. (2017). Integration of active videogames in physical training of school students. Scienceand Education, 4, 14-20.

Cuberos, R. C., Sánchez, M. C., O rtega, F. Z., Garcés, T. E. \& \& Martínez,A. M. (2016).Videojuegosactivoscomo recursoTIC en el auladeEducación Física: estudio a partir deparámetros de ocio digital-ActiveVideogames as ICT tool in Physical Education classroom: research from digital leisure parameters. Digital Education Review, 29, 112-123.

Elmore, G. M., \& Huebner, E. S. (2010). Adolescents' satisfaction with school experiences: Relationshipswith demographics, attachment rel ationships, and school engagement behavior. Psychology in the Schools, 47(6), 525-537. https: / / doi.org/ 10.1002/ pits. 20488

Escamilla Fajardo, P., N úñez-Pomar, J. M ., Ratten,V., \& Crespo, J. (2020). Entrepreneurship and Innovation in Soccer:Web of ScienceBibliometricA nalysis. Sustainability, 12 (11), 4499-4516. https:/ / doi.org/ 10.3390/ su12114499

FernandezAbuin, J. P. (2008). The O lympic M ovement and the new technologies in the area of Physical Education and Sport to inclination of the didactic pattern of webquest. Revista Internacional deM edicina y Cienciasdela Actividad Fisica y del D eporte, 8(29), 1-14. https:/ / doi.org/ 10.15366/ rimcafd

Flores, H. , Hui, P., Tarkoma, S., Li, Y., Srirama, S., \& Buyya, R. (2015). Mobilecodeoffloading: from concept to practiceand beyond. IEEE Communications J ournal, 53(3), 80-88. https:/ / doi.org/ 10.1109/ mcom.2015.7060486

Gao, P., Zhao, J., \& Li, X. (2019). Keyword Co-occurrenceAnalysis of Research on Innovation Ecosystem Studies. Economic Management Journal, 8(2), 25-36.

Gil, J. F. L., Cavichiolli, F. R., \& Lucas, J. L.Y. (2020). Programasde intervención paralapromoción de hábitos alimenticiossaludablesen escolaresespañoles practicantes de Educación Física: una revisión sistemática. Retos: nuevastendenciasen educación física, deportey recreación, (37), 786-792.

Göktaํㅡ. Z. (2018). TheAttitudes of Physical Education and Sport Students towards Information and Communication Technologies, TechTrends, 56(2), 22-30. https:/ / doi. org/ 10.1007/ s11528-012-0560-x

FilgueiraGomis,J. M . F. (2016). AppsParaAdquisición deH ábitos Saludables Dentro de laEducación Física. Revista deEducación Físca: Renovar LaTeoría y Practica, 144, 22-31. https:/ / doi.org/ 10.36443/10259/ 5451

Guan, A., Gao, C., Zhang, T., \& Zhou,W. (2018). Exploring the metaphor of information under the context of information scienceresearch: aco-wordsanalysis. Procedia Computer Science, 131, 739- 745. https// doi.org/ 10.1016/ j. procs. 2018.04.319 
Hinojo Lucena, F. J., Lopez Belmonte, J. , Fuentes Cabrera, A., TrujilloTorres, J. M., \& Pozo Sanchez, S. (2020). A cademic effects of the use of flipped learning in physical education. International journal of environmental research and public health, 17(1), 276. https:/ / doi.org/ 10.3390/ ijerph17010276

Hinojo-Lucena, F. . , M ingorance-Estrada, Á. C., Trujillo-Torres, J. M.,Aznar-Díaz, I. , \& Cáceres Reche, M. P. (2018). Incidence of the flipped classroom in the physical education students' academic performance in university contexts. Sustainability, 10(5), 1334-1346. https:/ / doi.org/ 10.3390/ su10051334

Kretschmann, R. (2015). Physical EducationTeachers' Subjective Theories about Integrating Information and Communication Technology (ICT) into Physical Education. Turkish Online ournal of Educational Technology-T0JET, 14(1), 68-96.

Legrain, P. , Gillet, N., Gernigon, C. , \& Lafreniere, M.-A. (2015). Integration of information and communication technology and pupils' motivation in a physical education setting. Journal of Teaching in Physical Education, 34(3), 384-401. https:/ / doi. org/ 10.1123/ jtpe. 2014-0013

León, M. P., Ayuso, A. P. \& M adrona, P. G. (2020). Hábitos y motivos de ejercicio físico en estudiantes universitariosy su relación con el valor otorgado alaEducación Física. Retos: nue vastendencias en educación física, deportey recreación, (37), 7884.

Meza, E. I.A., \& López, J.A. H. (2021). Physical activity in university student athletes, prior and in confinement due to pandemic associated with COVID-19. Retos: nuevastendencias en educación física, deportey recreación, (39), 112, 572 - 575.

Middelweerd, A., Mollee, J. S., van derWal, C. N., Brug, J., \&Te Velde, S. J. (2014). A ppsto promotephysical activity among adults: a review and content analysis. International Journal of Behavioral N utrition and Physical Activity, 11(1), 97-108. https: / / doi.org/ 10.1186/ s12966-014-0097-9

N ationalAssociation for Sportand Physical Education (2004). M oving into the future: National standardsfor physical education (2nd ed.). Columbus, OH: McGraw-Hill.

National Association for Sport and Physical Education. (2005). I sit physical education or physical activity? N ASPE position statement. Strategies, 19(2): 33-34.

O kely, A. D. , Booth, M. L. , \& Patterson, J.W. (2001). Relationship of physical activity to fundamental movement skills among adolescents. M edicineand Science in Sportsand Exercise, 33(11), 1899-1904. https/ / doi.org/ 10.1097/ 00005768-20011100000015

Panadero-Pérez, N., Román, R.-S. , Joya-Barroso, M. , \& Román$M$ ata, S. (2019). Digital sedentarism as a precursor to the deterioration of heal th adolescents and young people. ESH PA Education, Sport, H ealth and Physical Activity, 3(3), 368-383.

Papastergiou, M. (2009). Exploring the potential of computer and video games for heal th and physical education: A literature review. Computers \& Education, 53(3), 603-622. https:/ / doi.org/10.1016/ j.compedu.2009.04.001

Papastergiou, M. (2010). Enhancing physical education and sport science students' self-efficacy and attitudes regarding information and communication technologies through a computer literacy course. Computers \& Education, 54 (1), 298-
308. https: / / doi. org/ 10.1016/ j.compedu.2009.08.015

Prensky, M. (2001). Digital natives, digital immigrants. On the Horizon, $9(5)$.

RamosEchazarreta, R. ,ValdemorosSan Emeterio, M. Á. , SanzArazuri, E., \& Poncede LeónElizondo,A. (2011). Lainfluencia delosprofesoressobreel ocio físico deportivo delosjóvenes: Percepción de los agentes educativos más cercanos a ellos. Revista de Currículum y Formación de Profesorado, 11(2), 1-19. https:/ / doi.org/ 10.18172/ con.583

Skute, I. (2019). O pening the black box of academic entrepreneurship: abibliometric analysis. Scientometrics, 120(1), 237-265. https: / / doi. org/ 10.1007/ s11192-019-03116-w

Stanescu, M. , Stoicescu, M. , Bejan, R. , \&Vasiliu, . A. M. (2011). Computer use in physical education and sports teaching. ELearning \& Softwarefor Education, 11(5), 540-545.

Tearle, P. \& \& Golder, G. (2008). The use of ICT in theteaching and learning of physical education in compul sory education: how do we preparethe workforce of the future? European Journal ofTeacher Education, 31(1), 55- 72. https: / / doi.org/ 10.1080/ 02619760701845016

Teo, T. (2008). Pre-ser vice teachers' attitudestowards computer use: A Singapore survey. Australasian Journal of Educational Technology, 24(4), 413-424. https:/ / doi.org/ 10.14742/ ajet. 1201

Thomas, A. \& Stratton, G. (2006).W hat weare really doing with ICT in physical education: anational audit of equipment, use, teacher attitudes, support, and training British Journal of Educational Technology, 37(4), 617-632. https:/ / doi.org/ $10.1111 /$ j.1467-8535.2006.00520.x

Voogt, J., \& Knezek, G. (2008). International handbook of information technology in primary and secondary education (Vol. 20). Springer Science \& Business M edia.

Wyant, J., \& Baek, J.-H . (2019). Re-thinking technology adoption in physical education. Curriculum Studies in H ealth and Physical Education, 10(1), 3-17. https:/ / doi. org/ 10.1080/ 25742981.2018.1514983

Yang, L., Chen, Z., Liu, T., Gong, Z., Yu,Y., \&Wang, J. (2013). Global trends of solid waste research from 1997 to 2011 by using bibliometric analysis. Scientometrics, $96(1), 133-146$. https: / / doi.org/ 10.1007/ s11192-012-0911-6

Yerdelen-Damar, S. , Boz,Y., \&Ayd1n-Günbatar, S. (2017). M ediated effects of technology competenciesand experienceson relations among attitudes towards technology use, technology ownership, and self efficacy about technological pedagogical content knowledge. Journal of Science Education and Technology, 26(4), 394-405. https:/ / doi.org/ 10.1007/ s10956017-9687-z

Zurita-O rtega, F., Chacón-Cuberos, R., C astro-Sánchez, M., Gutiérrez-Vela, F. L. , \& González-Valero, G. (2018). Effect of an inter vention program based on active video games and motor games on health indicators in university students: A pilot study. International Journal of Environmental Research and Public H ealth, 15(7), 1329-1341. https:/ / doi.org/ 10.3390/ ijerph15071329 